\title{
Luttinger liquid superlattices
}

\author{
J. Silva-Valencia ${ }^{(1)}$, E. Miranda ${ }^{(1)}$, and Raimundo R. dos $\operatorname{Santos}^{(2)}$ \\ (1) Instituto de Física "Gleb Wataghin", Unicamp, C.P. 6165, 13083-970 Campinas SP, Brazil \\ ${ }^{(2)}$ Instituto de Física, Universidade Federal do Rio de Janeiro, C.P. 68.528, 21945-970 Rio de Janeiro RJ, Brazil
}

(October 30, 2018)

\begin{abstract}
We calculate the correlation functions and the DC conductivity of Luttinger liquid superlattices, modeled by a repeated pattern of interacting and free Luttinger liquids. In a specific realization, where the interacting subsystem is a Hubbard chain, the system exhibits a rich phase diagram with four different phases: two metals and two compressible insulators. In general, we find that the effective low energy description amalgamates features of both types of liquids in proportion to their spatial extent, suggesting the interesting possibility of 'engineered' Luttinger liquids.
\end{abstract}

PACS Nos. 71.10.Pm, 71.10.Fd, 71.30.+h, 73.20.Dx, 73.61.-r

In recent years, new experimental techniques have made it possible to grow nanostructures which are topologically one-dimensional, such as quantum wires and Carbon nanotubes. However, care must be taken when invoking existing models to discuss their electronic properties, since inhomogeneities must be taken into account in a fundamental way. Consider, for instance, the Luttinger liquid (LL), which is the standard model for lowenergy phenomena involving interacting electrons in one dimension [1]. The absence of conductance renormalization in long high-mobility GaAs wires [2] has been explained in terms of a usual LL (representing the wire) in contact with a non-interacting LL at each of its ends (representing the Fermi liquid leads); that is, overall, the system can be thought of as an inhomogeneous Luttinger Liquid (ILL). LL's with different inhomogeneity profiles have also been used in the context of the fractional quantum Hall effect (FQHE), to describe transitions between edge states at different fillings [3, 4, or between an edge state connected to a Fermi liquid [5]. A different class of inhomogeneous systems is represented by superlattices (SL's) and multilayers. By varying the relative thicknesses of the repeating unit in magnetic metallic multilayers, fascinating properties such as exchange oscillation and giant magneto-resistance (GMR) have been found [6]. The interplay between electron correlations and a SL structure (or layering) can therefore lead to collective properties quite distinct from those of each of its constituents. Furthermore, the ability to manipulate physical properties by choosing an appropriate spatial modulation opens the way for a whole new set of 'engineered' materials.

With this in mind, our purpose here is to discuss the properties of a one-dimensional SL made up of a periodic arrangement of two long and perfectly connected LL's, one interacting and the other free. Accordingly, the low-energy properties of this Luttinger liquid superlattice (LLSL) are described by generalizing the usual bosonized Hamiltonian [1, 7, 8] as follows

$$
H=\frac{1}{2 \pi} \sum_{\nu=\rho, \sigma} \int d x\left\{u_{\nu}(x) K_{\nu}(x)\left(\partial_{x} \Theta_{\nu}\right)^{2}\right.
$$

$$
\left.+\frac{u_{\nu}(x)}{K_{\nu}(x)}\left(\partial_{x} \Phi_{\nu}\right)^{2}\right\}
$$

where the sum extends over separated charge- $(\nu=\rho)$ and spin- $(\nu=\sigma)$ degrees of freedom, each of which with layer-dependent parameters $u_{\nu}(x)$ and $K_{\nu}(x)$; these determine, respectively, the velocity of elementary excitations and the algebraic decay of correlations functions for each degree of freedom. For $x$ on the free layer one has $K_{\nu}(x)=1$ and $u_{\nu}(x)=v_{F}$, the Fermi velocity, whereas for $x$ on the repulsive layer $K_{\nu}(x)$ and $u_{\nu}(x)$ become the usual uniform LL parameters. For definiteness, we will often speak of a 'Hubbard superlattice' (HSL), where the interacting layer is taken as a Hubbard model with hopping $t$ and on-site repulsion $U$ [9, 10]; a weak coupling perturbation theory similar to that of the homogeneous model can be used to show that Eq. (11) indeed describes the low energy and small momentum sector of the discrete model with long layers [11. In the homogeneous case, the dependence of the LL parameters on both the density and $U$ has been determined by recourse to the exact solution 12 15]. With respect to magnetic properties, the SL structure does not break spin SU(2) symmetry, so that the inhomogeneous $K_{\sigma}$ is still expected to renormalize to $K_{\sigma}^{*}=1$. The spin sector stiffness is therefore unrenormalized as in the homogeneous system [16.

The boson phase fields $\Phi_{\nu}$ are related to the charge and spin densities, $\rho$ and $\sigma$, through $\sqrt{2} \partial_{x} \Phi_{\nu}(x) / \pi=\nu$, while $\Theta_{\nu}$ is such that $\partial_{x} \Theta_{\nu}$ is the momentum field conjugate to $\Phi_{\nu}:\left[\Phi_{\nu}(x), \partial_{y} \Theta_{\nu^{\prime}}(y)\right]=i \delta_{\nu, \nu^{\prime}} \delta(x-y) . \Phi_{\nu}$ and $\Theta_{\nu}$ are dual fields, since they satisfy both

$$
\partial_{t} \Phi_{\nu}=u_{\nu}(x) K_{\nu}(x) \partial_{x} \Theta_{\nu}
$$

and the equation obtained through the replacements $\Phi_{\nu} \rightarrow \Theta_{\nu}, \Theta_{\nu} \rightarrow \Phi_{\nu}$, and $K_{\nu} \rightarrow 1 / K_{\nu}$. These equations can be uncoupled to yield

$$
\partial_{t t} \Phi_{\nu}-u_{\nu} K_{\nu} \partial_{x}\left(\frac{u_{\nu}}{K_{\nu}} \partial_{x} \Phi_{\nu}\right)=0
$$

and a dual equation for $\Theta_{\nu}$. 
The equations of motion are subject to the continuity of $\Phi_{\nu}$ and $\Theta_{\nu}$ 17, 18, (which ensures the continuity of the fermionic field). Since their time derivatives are also continuous, Eq. (2) and its dual yield, as additional conditions, the continuity of $\left(u_{\nu} / K_{\nu}\right) \partial_{x} \Phi_{\nu}$ and $u_{\nu} K_{\nu} \partial_{x} \Theta_{\nu}$ at the contacts. Thus, both charge and spin currents $j_{\nu}=\sqrt{2} \partial_{t} \Phi_{\nu} / \pi$ are conserved, since we neglect Umklapp processes and spin backscattering.

The Hamiltonian (1) is straightforwardly diagonalized by a normal mode expansion

$$
\begin{aligned}
\Phi_{\nu}(x, t)= & -i \sum_{p \neq 0} \operatorname{sgn}(p) \frac{\phi_{p, \nu}(x)}{2 \sqrt{\omega_{p, \nu}}}\left[b_{-p, \nu} e^{i \omega_{p, \nu} t}+b_{p, \nu}^{\dagger} e^{-i \omega_{p, \nu} t}\right] \\
& -\phi_{0, \nu}(x)+\gamma_{\lambda \nu} t \\
\Theta_{\nu}(x, t)= & i \sum_{p \neq 0} \frac{\theta_{p, \nu}(x)}{2 \sqrt{\omega_{p, \nu}}}\left[b_{-p, \nu} e^{i \omega_{p, \nu} t}-b_{p, \nu}^{\dagger} e^{-i \omega_{p, \nu} t}\right] \\
& +\theta_{0, \nu}(x)-\tau_{\lambda \nu} t
\end{aligned}
$$

where $b_{p, \nu}^{\dagger}$ are boson creation operators $(p>0) . \phi_{0, \nu}(x)$ and $\theta_{0, \nu}(x)$ are the zero mode functions which, in the homogeneous case, are given by $\phi_{0, \nu}(x)=N_{\nu} \frac{\pi x}{L}$, $\theta_{0, \nu}(x)=J_{\nu} \frac{\pi x}{L}$, where $N_{\nu}$ and $J_{\nu}$ are the (charge and spin) number and current operators. Besides, in this case $\gamma_{\nu}=\pi u_{\nu} K_{\nu} J_{\nu} / L$ and $\tau_{\nu}=\pi\left(u_{\nu} / K_{\nu}\right) N_{\nu} / L$. However, in a LLSL the inhomogeneity will induce a modulation of the charge (but not of the spin) density of the system. Thus, one needs to introduce in general layerspecific number and current operators. Since each layer is a LL, the variations across it are $\Delta \phi_{0, \nu}=\pi N_{\lambda \nu}$ and $\Delta \theta_{0, \nu}=\pi J_{\lambda \nu}$, where $\lambda=0$ or $U$, depending on whether it is a free or interacting layer, respectively. $\phi_{0, \nu}(x)$ and $\theta_{0, \nu}(x)$ will then be linear continuous functions of $x$, with slopes given by the layer number and current operators (we omit the expressions for brevity). Analogously, from the equations of motion (2), we obtain $\gamma_{\lambda \nu}$ and $\tau_{\lambda \nu}$.

In order to find the equilibrium value of the density in each layer, one needs to equate their chemical potentials

$$
\mu_{0}\left(n_{0}\right)=\mu_{0}\left(n+\ell\left(n-n_{U}\right)\right)=\mu_{U}\left(n_{U}\right),
$$

where $n=N / L$ is the total electron density, $\ell=L_{U} / L_{0}$ and $\mu_{\lambda}$ and $n_{\lambda}$ are the chemical potential and density of each layer, respectively. For definiteness, we have determined the charge profile in a HSL using the exact expression for $\mu_{U}\left(n_{U}\right)$ [13]. We found that the charge tends to accumulate in the free layer. This is rather intuitive, since electrons decrease their mutual repulsion energy by flowing into the free layer. This was observed in numerical studies of the HSL 10]. Of course, such a charge inhomogeneity will be strongly suppressed with the inclusion of long-range Coulomb interactions, which are absent in a Hubbard model description.

The HSL has a very rich phase diagram. For $n<1$ the system is always metallic. For $n>1$, however, we observe four different phases, two metallic and two insulating, each characterized by its charge profile, as shown

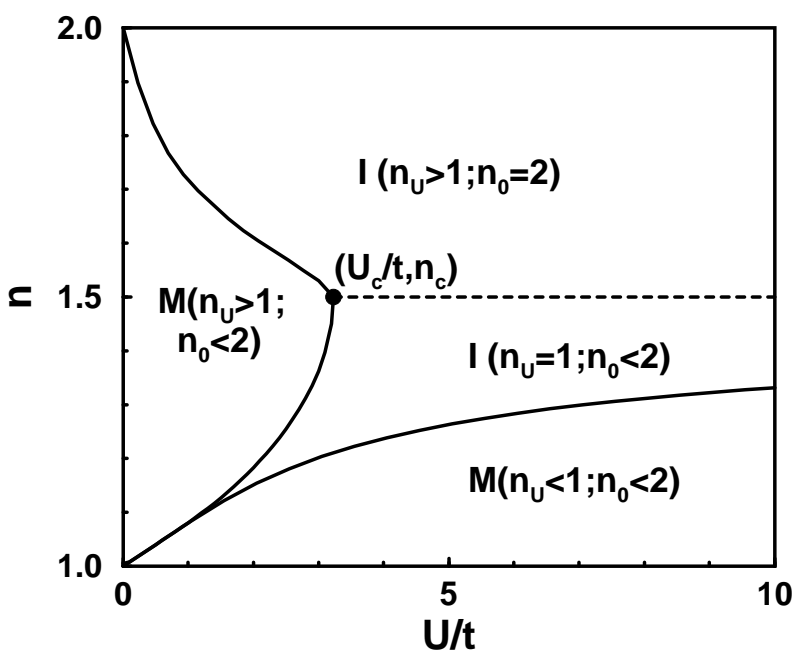

FIG. 1. Phase diagram of a Hubbard superlattice showing two metallic $(\mathrm{M})$ and two insulating $(\mathrm{I})$ phases $(\ell=1)$ $\left(U_{c} / t \approx 3.2309\right.$ and $\left.n_{c}=(2+\ell) /(1+\ell)\right)$.

in Fig. 11. The two insulating phases correspond to either $n_{U}=1$ (Mott insulator) or $n_{0}=2$ (band insulator). Note that, in each case, one type of layer is by itself insulating while the other is metallic, the overall insulating character being a consequence of the 1D structure ('resistors in series'). Therefore, both insulating phases are gapless, except at the phase boundary indicated by the dashed line in Fig. 1, where both $n_{U}=1$ and $n_{0}=2$ and the system exhibits a Mott-Hubbard gap. The density at this line is thus $n_{c}=(2+\ell) /(1+\ell)$ 19. The two metallic phases differ in the density of the interacting layer: the chemical potential can fall in either its upper or its lower Hubbard band. For values of $U>U_{c} \approx 3.2309 t$, the system cannot sustain the upper metallic phase. This value is independent of the 'aspect ratio' $\ell$. Note that, for $U<U_{c}$, the HSL is always gapless.

While the topology of the phase diagram of Fig. 1 is specific to a HSL, we expect that several of its features should be generic to other LLSL's. In particular, the 'division of labor' between the two subsystems, where one is responsible for the insulating behavior whereas the other renders the system compressible, is reflected in the weighted form of the SL compressibility

$$
\kappa_{s}=\frac{1}{L}\left(\frac{\partial^{2} E_{0}}{\partial N^{2}}\right)^{-1}=\frac{\kappa_{0}+\ell \kappa_{U}}{1+\ell},
$$

where $\kappa_{U}=2 K_{\rho} / \pi u_{\rho}$ and $\kappa_{0}=2 / \pi v_{F}$ are the compressibilities of the interacting and free layers respectively.

The SL structure also affects the velocity of excitations. For $p \ll \pi /\left(L_{U}+L_{0}\right)$, the dispersion relation of the LLSL is linear, with effective velocities

$$
c_{\nu}=\frac{v_{F}(1+\ell)}{\sqrt{1+\Delta_{\nu} \ell v_{F} / u_{\nu}+\left(\ell v_{F} / u_{\nu}\right)^{2}}}
$$


where $\Delta_{\nu}=K_{\nu}+K_{\nu}^{-1}$. Clearly, $c_{\nu} \rightarrow u_{\nu}$ as $\ell \rightarrow \infty$, and $c_{\nu} \rightarrow v_{F}$ as $\ell \rightarrow 0$. Furthermore, as one approaches the insulating phase from the low-density region (see Fig. 1), $c_{\rho} \rightarrow 0$ as a result of $u_{\rho} \rightarrow 0$ in the interacting layer. As in the homogeneous system, the LL description breaks down whenever a gap opens in the charge or spin sector of either layer. In the HSL case, this happens in both insulating regions of Fig. 1. Note, however, that the determination of the phases through Eq. (6) does not rely on the LL description.

We now focus on the correlations. The $T=0$ asymptotic behavior (i.e., for well separated $x$ and $y$ ) of charge and spin correlations is given by

$$
\begin{aligned}
\langle n(x) n(y)\rangle \sim & \frac{\alpha_{\rho}}{\pi^{2}|x-y|^{2}}+A_{1} \frac{e^{2 i\left(\bar{\phi}_{0}(y)-\bar{\phi}_{0}(x)\right)}}{|x-y|^{1+K_{\rho}^{*}}} \\
& +A_{2} \frac{e^{4 i\left(\bar{\phi}_{0}(y)-\bar{\phi}_{0}(x)\right)}}{|x-y|^{4 K_{\rho}^{*}}}, \\
\langle\mathbf{S}(x) . \mathbf{S}(y)\rangle \sim & \frac{\alpha_{\sigma}}{\pi^{2}|x-y|^{2}}+B_{1} \frac{e^{2 i\left(\bar{\phi}_{0}(y)-\bar{\phi}_{0}(x)\right)}}{|x-y|^{1+K_{\rho}^{*}}},
\end{aligned}
$$

where $\bar{\phi}_{0}(x)=k_{F} x-\phi_{0, \rho}(x)$ and the LLSL effective exponent is

$$
K_{\rho}^{*}=\frac{\sqrt{1+\Delta_{\rho} \ell v_{F} / u_{\rho}+\left(\ell v_{F} / u_{\rho}\right)^{2}}}{1+\ell v_{F} / K_{\rho} u_{\rho}} \equiv f\left(K_{\rho}\right),
$$

here $\alpha_{\nu}$ is a function of system parameters and the layer. Similarly, correlation functions for singlet and triplet superconducting pairing are

$$
\left\langle O^{\dagger}(x) O(y)\right\rangle \sim \frac{C}{|x-y|^{1+\bar{K}_{\rho}}},
$$

where $\bar{K}_{\rho}=f\left(1 / K_{\rho}\right)$. One should note that the correlation functions depend not only on the difference $x-y$, but also on the actual positions $x$ and $y$, through the zero mode functions. Their effect will be to generate the usual spatial oscillations present in homogeneous LL's. However, due to the inhomogeneous density profile, their period will vary from layer to layer, reflecting the layerdependent Fermi wave-vectors; this is akin to the oscillatory behavior of the exchange coupling in magnetic metallic multilayers 66,10$]$. In spite of the presence of effective exponents $K_{\rho}^{*}$ and $\bar{K}_{\rho}$, the condition for dominant superconducting correlations reduces to the one for homogeneous systems, namely $K_{\rho}>1$. The dominant term in the charge and spin correlation functions is the second one, which in the homogeneous case corresponds to the $2 k_{F}$ contribution. This predominance, however, may be superseded by the behavior of the amplitude $A_{1}$, as discussed in Ref. [20].

In Fig. 2, the correlation exponent $K_{\rho}^{*}$ of a HSL is shown as a function of filling, for $\ell=1$. Both metallic

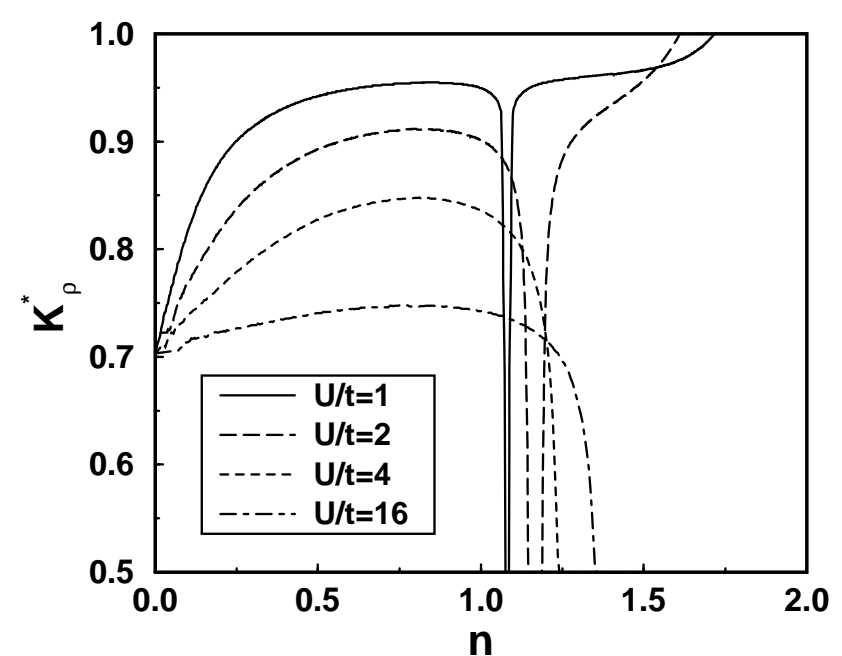

FIG. 2. The correlation exponent $K_{\rho}^{*}$ of a Hubbard superlattice as a function of the total electron density $n$ for $\ell=1$ and different values of $U$.

phases are characterized by $1 / 2<K_{\rho}^{*}<1$. On the low density side, $K_{\rho}^{*}$ approaches a value larger than $1 / 2$ as $n \rightarrow 0$, which depends on $\ell$ but not on $U$. This is a feature unique to the LLSL. Note from Eq. (11) that $K_{\rho}^{*}$ interpolates monotonically between 1 (the free value) and $K_{\rho}$ (the interacting layer exponent) as $\ell$ is varied from 0 to $\infty$. This illustrates a general feature of the LLSL, namely: by varying the 'aspect ratio' $\ell$, one can fine-tune a physical property to a specified value.

Finally, we discuss the transport properties of a LLSL. In the presence of an applied electric field the equation of motion for $\Phi_{\rho}$ becomes 17,18

$$
\left[-\frac{\partial_{t t}}{u_{\rho} K_{\rho}}+\partial_{x}\left(\frac{u_{\rho}}{K_{\rho}} \partial_{x}\right)\right] \Phi_{\rho}(x, t)=-e E(x, t) .
$$

The nonlocal conductivity is given by

$$
\sigma(x, y, t)=-\frac{2 g_{o}}{\pi} \partial_{t} G(x, y, t)
$$

where $g_{o}=e^{2} / h$ is the conductance quantum and $G(x, y, t)=-i \theta(t)\left\langle\left[\Phi_{\rho}(x, t), \Phi_{\rho}(y, 0)\right]\right\rangle$ is the bosonic Green's function.

We first calculate the Drude conductivity, which gives the current response to a uniform electric field: $\lim _{\omega \rightarrow 0} \sigma(q=0, \omega)$ [21]. A straightforward calculation yields

$$
\sigma(q=0, \omega)=2 g_{o} c_{\rho} K_{\rho}^{*} \delta(\omega),
$$

The delta function coefficient is the Drude weight. It has the same form as for the homogeneous case [12], but with the effective velocity and effective exponent replacing the corresponding uniform quantities $u_{\rho}$ and $K_{\rho}$. By plugging in the results from Eqs. (8) and (11), one recognizes the conductivity of resistors connected in series. 


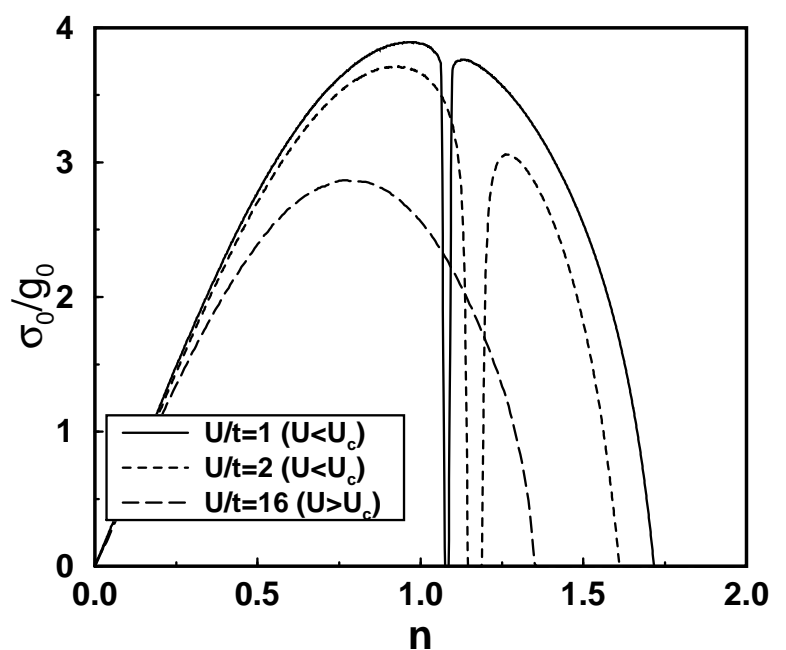

FIG. 3. The Drude weight of a Hubbard superlattice as a function of $n$ for $\ell=1$ and different values of $U$.

We have plotted the Drude weight of a HSL as a function of $n$ for $\ell=1$ and several values of $U$ in Fig. 3. The plot shows the re-entrant behavior as a function of $n$ for $U<U_{c}$. Furthermore, the Drude weight dips to zero upon approaching the insulating regions as a result of the vanishing charge velocities $u_{\rho} \rightarrow 0$ (Mott insulator) and $v_{F} \rightarrow 0$ (band insulator).

A more common experimental situation occurs when a field is applied to a finite region of the sample. In this case, the inverse order of limits applies $\lim _{q \rightarrow 0} \sigma(q, \omega=$ 0 ), and we obtain the Landauer conductance [21]. In the LLSL

$$
\sigma(q, \omega=0)=2 g_{o} K_{\rho}^{*} \delta(q) .
$$

Once again, the result is in close analogy with the homogeneous system [22], where the SL interaction exponent has replaced the homogeneous one. Both Drude and Landauer responses, therefore, can be modulated by changing the SL spacing. However, the interaction renormalization of Eq. (16) is not revealed in usual DC conductance measurements, where it is masked by the presence of the Fermi liquid leads [17]. In a LLSL of length $L$, only by going to frequencies of the order of the inverse traversal time $\omega>u_{\rho} / L$ can the influence of the $K_{\rho}^{*}$ exponent be felt [23].

In closing, we would like to highlight the fact that the low energy, long wavelength properties of a LLSL can be, in effect, subsumed into a few effective parameters, in close analogy with the usual homogeneous LL description. These effective parameters, on the other hand, turn out to be weighted averages of the underlying subsystem properties, in rough proportion to their spatial extent [see, e.g., Eqs. (7), (8) and (11)]. Such a 'tempered Luttinger liquid' description suggests the interesting possibility of creating SL structures with properties engineered to suit a particular purpose, in a way reminiscent of modulation-doped semiconductor heterostructures and magnetic multilayers. Whether this will prove feasible, however, remains to be seen.

In summary, we have considered Luttinger liquid superlattices made up of a periodic arrangement of free and repulsive Luttinger liquids. Due to the space-dependent properties of the system, a non-homogeneous charge profile ensues. A specific realization of such a system, a Hubbard superlattice, was investigated in detail and its phase diagram was shown to exhibit two metallic phases and two peculiar compressible insulating ones.

The authors are grateful to A O Caldeira and $\mathrm{T}$ Paiva for discussions. Financial support from the Brazilian Agencies CNPq, FAPESP (E.M.), and FAPERJ (R.R.d.S.) is also gratefully acknowledged.

[1] J. Voit, Rep. Prog. Phys. 57, 977 (1994).

[2] S. Tarucha, T. Honda, and T. Saku, Solid State Commun. 94, 413(1995).

[3] Y. Oreg and A. M. Finkel'stein, Phys. Rev. Lett. 74, 3668 (1993).

[4] D. B. Chklovskii and B. Halperin, Phys. Rev. B 57, 3781 (1998).

[5] C. de C. Chamon and E. Fradkin, Phys. Rev. B 56, 2012 (1997).

[6] M. N. Baibich and R. B. Muniz, Braz. Journ. Phys. 22, 253 (1992); B. Heinrich and J. F. Cochran, Adv. Phys. 42, 523 (1993); K. B. Hathaway, in Ultrathin Magnetic Structures II, edited by B. Heinrich and J. A. C. Bland, (Springer, Berlin, 1994).

[7] V. J. Emery, in Highly Conducting One-dimensional Solids, edited by J. T. Devreese, R. P. Evrard, and V. E. Van Doren (Plenum, New York, 1979) p. 247.

[8] F. D. M. Haldane, J. Phys. C 14, 2585 (1981).

[9] T. Paiva and R. R. dos Santos, Phys. Rev. Lett. 76, 1126 (1996).

[10] T. Paiva and R. R. dos Santos, Phys. Rev. B 62, 7007 (2000).

[11] I. Safi, Ann. Phys. (Paris) 22, 463 (1997).

[12] H. J. Schulz, Phys. Rev. Lett. 64, 2831 (1990); Int. Jour. Mod. Phys. B 5, 57 (1991).

[13] E. H. Lieb and F. Y. Wu, Phys. Rev. Lett. 20, 1445 (1968).

[14] H. Frahm and V. E. Korepin, Phys. Rev. B 42, 10553 (1990).

[15] N. Kawakami and S. K. Yang, Phys. Lett. A 148, 359 (1990).

[16] However, a gap has been predicted in HSL's with an even number of electrons on short repulsive layers [9,10].

[17] I. Safi and H. J. Schulz, Phys. Rev. B 52, R17040 (1995); D. L. Maslov and M. Stone Phys. Rev. B 52, R5539 (1995); V. V. Ponomarenko, Phys. Rev. B 52, R8666 (1995). 
[18] I. Safi and H. J. Schulz, Phys. Rev. B 59, 3040 (1999).

[19] T. Paiva and R. R. dos Santos, Phys. Rev. B 58, 9607 (1998).

[20] T. Paiva and R. R. dos Santos, Phys. Rev. B 61, 13480 (2000).

[21] E. W. Fenton, Phys. Rev. B 46, 3754 (1992).

[22] W. Apel and T. M. Rice, Phys. Rev. B 26, 7063 (1982).

[23] K. A. Matveev and L. I. Glazman, Physica B 189, 266 (1993). 